\title{
EXAMINATION RESULTS
}

Examination for Fellowship of the Royal College of Physicians of Canada MODIFIED FOR ANAESTHESIA

Bhavakula, Dr. K., Toronto, Ont. BrtTt, Dr. B.A., Toronto, Ont. Catron, DR. D. V., Hamilton, Ont. CervenKo, Dr. F. W., London W.C., Eng.

Einarson, Dr. D. W., Kingston, Ont. Fatrhunst, Dr. K. W., Toronto, Ont. Houle, Dr. G. L., Pointe Claire, P.Q. James, Dr. W. A., Don Mills, Ont. LABRECQUE, Dr. B., Montreal, P.Q. LA Pointe, Dr. A., Jonquiére, P.Q.

LOUNDER, DR. J. H., Canadian Forces, Europe
McFarLAND, Dr. J. D., Saskatoon, Sask.

McIntosh, Dr. R. W., Don Mills, Ont. Pryapongse, Dr. M., Toronto, Ont. ReNSAA, Dr. M. A., Edmonton, Alta. Sindon, Dr. A., Ville d'Anjou, Montréal, P.Q.

SYSLAR, Dr. W. B., Winnipeg, Man. TURNer, DR. M. R., Vancouver, B.C. WeBer, Dr. R. K., Toronto, Ont.

\section{Examination for Certiftcation in Anaesthesta}

Arranz, Dr. C., Riverview, N.B.

Bhambhant, Dr. M. D., Montréal, P.Q.

Bisson, Dr. L., Trois-Rivières, P.Q.

Busque, Dr, L., Ste-Foy, P.Q.

Campbelt, Dr. N. J., Vancouver, B.C.

Carballo, Dr. A. S., Burnaby, B.C.

Custeau, Dr. P., St-Léonard, Montréal, P.Q.

Dagenais, Dr. G., Montréal, P.Q. DE JoNGH, DR. H. J., Edmonton, Alberta

Desnoyers, Dr. Y., Ville d'Anjou, Québec, P.Q.

Desrosiers, Dr. R., Ste-Foy, Québec, P.Q.

Dixon, Dr. M. J., Montréal, P.Q.

Dube, Dr. Y., Alma (Cté Lac St.-Jean), P.Q.

Ethans, Dr. C. T., Winnipeg, Man.

FIteAu, Dr. M., Ville Mont-Royal, P.Q.

Gagnon, Dr. D., Rivière-du-Loup, P.Q.
Greaves, Dr. P. C. W., Newmarket, Ont.

HaYNes, Dr. H. G., Edmonton, Alberta HunTER, Dr. D. D., Islington, Ont. KELLX, Dr. A. C., Lancaster, N.B.

Khan, Dr. I., Cote St-Luc, Montréal, P.Q.

KIM, Dr. J. H., Regina, Sask.

Krestow, Dr. M., Toronto, Ont.

LEACock, Dr. A. M., Edmonton, Alberta

LEFebvre, Dr, G., Montréal, P.Q. LEMIRE, DR, C., Montréal, P.Q.

LEwIs, Dr. M. J., Edmonton, Alberta Mastropasqua, Dr. C. A., N.D.G., Montreal, P.Q.

MaCutcheon, Dr. R. M., Toronto, Ont. McGregor, Dr. J. A., Regina, Sask. MCJANNET, DR. W. A., Halifax, N.S. McKeever, Dr. R. W., St. John's, Nfld. MIIIER, DR. J. D. M., Edmonton, Alberta

Mrtron, Dr. C. C., Pont-Viau, P.Q. 
Morse, Dr. A. L., Montréal, P.Q.

O'K GeFE, DR. A. J., Washington, U.S.A. Petrie, DR. E. A., JR., Fredericton, N.B. Pucr, Dr. R. F., Saskatoon, Sask. Rojko, Dr. A., Don Mills, Ont. Rousseau, Dr. P. J. J., Montréal, P.Q. TABESH, Dr. A. R., St. Boniface, Man. Thomson, DR. J. G., Winnipeg, Man.
Treissman, DR. D. A., Richmond Hill, Ont.

Vezina-Voyer, Dr. J., Beauceville-est, P.Q.

Wallat, DR. G. E., Calgary, Alberta Weston, DR. R. B., Willowdale, Ont. Wrseman, Dr. L. J., Cooksville, Ont. 